\title{
Oxidant and Antioxidant Balance in Children with Community-Acquired Pneumonia
}

\author{
Kubra Aykac ${ }^{1}$, yasemin ozsurekci ${ }^{2}$, Sevgen TANIR BASARANOGLU², Gamze Avcioglu ${ }^{3}$, \\ Eda Karadag Oncel ${ }^{2}$, Ozcan Erel $^{3}$, and Mehmet Ceyhan ${ }^{2}$ \\ ${ }^{1}$ Hacettepe Universitesi Tip Fakultesi \\ ${ }^{2}$ Hacettepe University Faculty of Medicine \\ ${ }^{3}$ Yildirim Beyazit University Faculty of Medicine
}

September 17, 2020

\begin{abstract}
Objective: There is a crucial balance between oxidant and antioxidant defense mechanisms. We aimed to evaluate the role of the balance of these systems in community-acquired pneumonia (CAP) in children. Methods: We analyzed serum oxidant and antioxidant stress parameters according to the clinical and demographic data of children with CAP and compared them with healthy controls. Serum total antioxidant status (TAS), total oxidant status (TOS), and levels of ischemia-modified albumin (IMA), antioxidant enzymes, non-enzymatic antioxidant factors, and plasma thiol were evaluated and compared between the groups. Results: Of 160 children evaluated, 106 had CAP (54 outpatients, 52 inpatients) and the other 54 were healthy subjects (control group). Total thiol and native thiol levels were significantly lower in the inpatient group compared to the outpatient group ( $\mathrm{p}=0.004, \mathrm{p}=0.005)$. Serum IMA differed significantly among the groups $(\mathrm{p}=0.001)$, with inpatients showing the highest level. A positive correlation was found between serum IMA and C-reactive protein levels in patients with pneumonia ( $\mathrm{r}=0.351$; $\mathrm{p}=0.001$ ). Conclusion: Parameters that provide information about antioxidant capacity may be useful in the diagnosis and prognosis of pneumonia. Both thiol homeostasis parameters and IMA level seem likely to be influenced by disease severity. Our results suggest that plasma thiol levels and IMA may be good candidate biomarkers to predict the severity of pneumonia in children.
\end{abstract}

\section{Running title: Oxidative Stress in Children with Pneumonia}

\section{Introduction}

Community-acquired pneumonia (CAP) is defined as a clinical diagnosis of pneumonia in a previously healthy child due to an infection acquired outside of the hospital ${ }^{1}$. CAP affects children and families worldwide and accounted for $15 \%$ of global deaths among children under 5 years of age in 2015 . While most healthy children can fight the infection using their natural defenses, children whose immune system is compromised are at higher risk of developing pneumonia ${ }^{2}$.

Oxidant stress has become a major topic in all areas of medicine. It can contribute to the pathogenesis of several lung diseases in children, such as asthma, acute bronchiolitis, cystic fibrosis, acute respiratory distress syndrome, and chronic neonatal lung disease ${ }^{3,4}$. In fact, there is a critical balance between oxidant and antioxidant defense mechanisms in the body, and oxidative stress is defined as an imbalance between the oxidant and antioxidant systems. Alteration of the oxidative metabolism is considered one of the leading pathogenic mechanisms in the development and progression of $\mathrm{CAP}^{5}$. The clinical manifestations of CAP vary significantly in severity. Diagnosis and estimating prognosis are critical for effectively managing patients with CAP. For this reason, several diagnostic and prognostic biomarkers have been investigated for CAP ${ }^{6}$. 
Due to the role of oxidative stress in the pathogenesis of CAP, we aimed to evaluate the role of the balance of these systems in the diagnosis and clinical course of CAP in children.

\section{Methods}

\section{Study population}

This prospective cross-sectional study was conducted at Hacettepe University Department of Pediatric Infectious Diseases in Turkey between 2016 and 2017. All children between 3 months and 18 years of age who presented during the study period and were diagnosed as having CAP were included in the study. CAP was defined as a clinical diagnosis of pneumonia caused by a community-acquired infection in previously healthy children 7. Children with CAP who had a temperature less than $38.5^{\circ} \mathrm{C}$, exhibited mild chest recession or dyspnea, and were taking full feeds with no vomiting were managed as outpatients. Children who had a temperature over $38.5^{\circ} \mathrm{C}$, tachypnea, moderate or severe chest recession, severe dyspnea, nasal flaring, cyanosis, intermittent apnea, grunting respiration, impaired feeding, tachycardia, capillary refill time [?]2 s were admitted and treated as inpatients ${ }^{1}$. A control group of healthy age- and sex-matched children were also enrolled in the study.

In total, 160 children were included and categorized into 3 groups: outpatient CAP, inpatient CAP, and health controls. The children's demographic and clinical data (including age, sex, weight, medical history, physical examination findings, risk factors, complications, and CAP severity) were collected and blood samples were collected for measurement of serum oxidant and antioxidant stress parameters.

\section{Laboratory tests}

Serum total antioxidant status (TAS), total oxidant status (TOS), oxidative stress index (OSI), levels of albumin, plasma thiol, disulphide, paraoxonase (PON), stimulated paraoxonase (SPON), catalase (CAT), myeloperoxidase (MPO), ceruloplasmin (CLP), ischemia-modified albumin (IMA), and C-reactive protein (CRP), arylesterase (ARES) activity, white blood cell (WBC) count, platelet count, and erythrocyte sedimentation rate (ESR) were evaluated and compared among the groups.

Blood samples were obtained from all CAP patients within the 48 hours of presentation and before initiating treatment. The whole blood was centrifuged at $1500 \mathrm{rpm}$ for 5 minutes and the serum was stored at $80 \mathrm{degC}$ until analysis. The serum samples were analyzed for oxidant and antioxidant stress parameters in the biochemical laboratory of Ataturk Training and Research Hospital in Ankara. Patients' hemogram, CRP, and ESR were measured at the time of enrollment to the study. In the healthy control group, blood samples were obtained when they presented to the outpatient clinic.

Thiol/disulphide levels were analyzed using a method developed by Erel and Neselioglu [8]. Serum TAS, TOS, and OSI were measured using automated colorimetric measurement methods as described by Erel ${ }^{9,}, 10,11$.

Paraoxonase assays were performed in the absence and presence of $\mathrm{NaCl}$ (salt-stimulated activity, SPON) 12. CAT activity was measured by Goth's method ${ }^{13}$. Measurement of ARES activity was performed using phenylacetate as the substrate ${ }^{14}$. MPO activity was measured by a modified O-dianisidine method, which is a kinetic measurement ${ }^{15}$. CLP level was measured using the method described by Erel ${ }^{16}$. Serum IMA levels were measured using the colorimetric assay method previously described by Bar-Or et al. ${ }^{17}$.

\section{Statistical analyses}

All statistical analyses were performed using SPSS version 19.0 (SPSS, Inc., Chicago, IL, USA) and Medcalc Version 18.6 statistical software. Descriptive statistics were used to summarize the participants' baseline characteristics, including mean, standard deviation (SD), median, and minimum-maximum values for continuous variables and frequency distributions for categorical variables. For comparisons of more than 2 groups, ANOVA was used for normally distributed continuous variables, while Kruskal-Wallis test was preferred for non-normally distributed variables. Multiple comparisons were made using the Games-Howell test or Mann-Whitney $U$ test with Bonferroni correction. Receiver operating characteristic (ROC) curve analysis was performed to determine cut-off values to distinguish between the study and control groups, and 
their sensitivity and specificity were calculated. In all analyses, the tests were two-tailed and $p<0.05$ was considered significant.

\section{Results}

A total of 160 children were evaluated; 106 had CAP, including 54 outpatients and 52 inpatients, and the other 54 children were healthy controls (Table 1 ). There were no statistically significant differences in age or gender between the groups ( $\mathrm{p}>0.05)$. In terms of medical history, $85.2 \%$ of the outpatients had no preceding illness, whereas only $32.7 \%$ of the inpatients were previously healthy. All of the children in the control group were healthy.

Median WBC count and CRP values differed significantly among the groups $(\mathrm{p}=0.02$ and $\mathrm{p}=0.001)$. The inpatient group had higher WBC count compared to the control group and higher CRP level than both of the other groups. The mean TAS values of the outpatient CAP, inpatient CAP, and control groups were 1.6+- 0.2 and $1.6+-0.3$, and $1.3+-0.2 \mathrm{mmol}$ Trolox equiv./L, respectively, with significantly higher level in the CAP patients compared to controls ( $\mathrm{p}=0.001)$. CAT level was significantly higher in the inpatient group compared to the outpatient and control groups $(205 \mathrm{kU} / \mathrm{L}, 163 \mathrm{kU} / \mathrm{L}$, and $99 \mathrm{kU} / \mathrm{L}$, respectively; $\mathrm{p}=0.003)$. The MPO, CLP, and ARES values of patients were lower in controls than outpatients with pneumonia $(\mathrm{p}=0.02, \mathrm{p}=0.02$, and $\mathrm{p}=0.03$, respectively). Mean serum IMA levels were $0.5+-0.1$ absorbance unit (ABSU) in outpatients, $0.7+-0.1$ ABSU in inpatients, and 0.6 +- 0.08 ABSU in the control group $(\mathrm{p}=0.001)$. Serum albumin level differed significantly between inpatients and outpatients $(\mathrm{p}=0.02)$. Although serum IMA level was higher among inpatients than the other groups, serum albumin level was lower in inpatients than outpatients (Figure 1). A positive correlation was found between serum IMA and CRP levels in patients with CAP ( $\mathrm{r}=0.351$; $\mathrm{p}=0.001)$.

Native thiol (NT) levels in the outpatient, inpatient, and control groups were $410 \mu \mathrm{mol} / \mathrm{L}, 346 \mu \mathrm{mol} / \mathrm{L}$, and $361 \mu \mathrm{mol} / \mathrm{L}$, respectively, while total thiol (TT) levels were $450 \mu \mathrm{mol} / \mathrm{L}, 392 \mu \mathrm{mol} / \mathrm{L}$, and $411 \mu \mathrm{mol} / \mathrm{L}$, respectively. TT and NT levels were significantly lower in the inpatient group than in the outpatient group $(\mathrm{p}=0.004, \mathrm{p}=0.005)$ but higher in the total CAP patient group compared to the control group.

The median TOS values in the outpatient, inpatient, and control groups were $10.5 \mu \mathrm{mol} \mathrm{H}_{2} \mathrm{O}_{2} / \mathrm{L}, 13.1 \mu \mathrm{mol}$ $\mathrm{H}_{2} \mathrm{O}_{2} / \mathrm{L}$, and $15 \mu \mathrm{mol} \mathrm{H}_{2} \mathrm{O}_{2} / \mathrm{L}$, respectively. There was no statistically significant difference in TOS among the groups $(\mathrm{p}=0.06)$. Likewise, the median ESR, platelet count, disulphide, PON, and SPON levels were not different significantly among the groups ( $\mathrm{p}>0.05$ for all).

WBC count, OSI, and levels of CRP, NT, TT, albumin, MPO, CLP, and IMA showed significant differences between the control and total CAP patient group (inpatients and outpatients) ( $\mathrm{p}<0.05$ for all). WBC, CRP, NT, TT, IMA, MPO, and CLP were higher in CAP patients than controls, while OSI and albumin were lower.

Optimal cut-off values for NT, TT, CRP, and IMA to predict hospital admission in children with CAP were identified by ROC curve analysis. The ROC curves of inpatients versus outpatients with CAP are shown in Figure 2. The optimal cutoff value of admitting NT level was $364 \mu \mathrm{mol} / \mathrm{L}$, which had a sensitivity of $60 \%$ and specificity of $76 \%$ (area under the curve $[\mathrm{AUC}]=0.67,95 \%$ confidence interval $[\mathrm{CI}]: 57-78 ; \mathrm{p}=0.002$ ). The optimal cutoff value of admitting TT level was $403 \mu \mathrm{mol} / \mathrm{L}$, which had a sensitivity of $58 \%$ and specificity of $74 \%$ (AUC $=0.67,95 \%$ CI: $57-78 ; \mathrm{p}=0.002$ ). The optimal cutoff value of admitting CRP level was 3.2 $\mathrm{mg} / \mathrm{L}$, which had a sensitivity of $65 \%$ and specificity of $84 \%$ (AUC $=0.73,95 \%$ CI: $55-91 ; \mathrm{p}=0.02$ ). The optimal cutoff value of admitting IMA was 0.6 ABSU, which had a sensitivity of $56 \%$ and specificity of $87 \%$ $(\mathrm{AUC}=0.61,95 \%$ CI: 52-70; $\mathrm{p}=0.01)$.

\section{Discussion}

This study analyses the prognostic value of oxidant and antioxidant parameters in children with CAP. Our results suggest that TT, NT, IMA, and CRP levels may be useful predictors of the severity of CAP in children. Decreases in TT and NT levels with increases in IMA and CRP levels may guide clinicians for accurate and early management of CAP, especially in children. 
In infectious diseases, various inflammatory cells are activated and reactive oxygen species (ROS) are produced ${ }^{18}$. In CAP, the balance between oxidant and antioxidant capacity shifts and ROS enhanced towards a response to primary host defense mechanism through phagocytes activation, immune cell functions, activation of inflammatory cascades and expression of adhesion molecules in $\mathrm{CAP}^{19}$. Thiols comprise the major portion of the total body antioxidants and play an important role against ROS, protecting against damage induced by free radicals ${ }^{20}$. Thiol levels decrease in order to neutralize ROS in case of high oxidative stress 21. There are many studies investigating thiol status in adults and children in different diseases including acute tonsillopharyngitis, meningism, meningitis, acute appendicitis, Crimean-Congo Hemorrhagic Fever (CCHF) and brucellosis ${ }^{18,22,23,24,25}$, but few studies have examined thiol status in patients with CAP 26, 27. Consistent with our results, previous studies found that inpatients had lower TT and NT levels compared with control groups 18, 22, 23, 25. Moreover, Aycicek et al. and Oncel et al. reported the same results in children as in our study ${ }^{24,25}$. However, our finding that outpatients had higher thiol levels supported the potential utility of thiol level as a predictor of CAP severity, because the higher level in outpatients may be an indirect indicator of improved antioxidant status in the lung tissue due to better control of the proinflammatory processes in the lungs ${ }^{27}$. In one of our previous studies focused on the oxidant status of patients with CCHF, Oncel et al. reported that high TT may be protective against the development of CCHF infection. In other words, similar to the literature, the progression of inflammatory processes cannot be inhibited without inadequate thiol levels, as in inpatients of our study ${ }^{22,23,24,25}$. In addition, Parlak et al. also concluded that the thiol/disulphide state may be an important variable in monitoring treatment response ${ }^{26}$.

Human albumin is a major circulating protein with antioxidant properties ${ }^{21}$. The N-terminal region of albumin binds transition metals such as cobalt, copper, and nickel. ROS damage the N-terminal region of albumin, thereby diminishing its metal-binding capacity. This modified form of albumin is called IMA, and its level increases with oxidative stress ${ }^{21,28}$. CRP is one of the most common biomarkers investigated in many kinds of infectious and inflammatory processes, and is a well-established biomarker in pneumonia ${ }^{1,29}$. It is known that CRP is a positive acute-phase protein (APP), whereas albumin is a negative APP due to reduced albumin synthesis by the liver ${ }^{30}$. Elevated serum IMA levels have been reported previously in infectious diseases such as neonatal sepsis and adult CAP ${ }^{31,32}$. In the present study, CRP and IMA levels were higher in inpatients with CAP than the other groups. In addition, we observed a positive correlation between serum IMA and CRP levels in patients with pneumonia, as reported by Bolatkale et al. ${ }^{31}$. These results may also be useful in the prediction of disease severity in pediatric patients with CAP.

Many studies have shown that oxidative stress contributes to the pathogenesis of several lung diseases, including asthma, cystic fibrosis, lung cancer, acute respiratory lung injury, chronic obstructive lung disease, and pneumonia ${ }^{3,33}$. Muravlyova et al. found that there was fix elevation of oxidative proteins in erythrocytes and higher concentrations of malondialdehyde in CAP patients than in controls ${ }^{5}$. Trefler et al. observed higher oxidative stress in CAP patients compared to healthy volunteers ${ }^{34}$. Katsoulis et al. showed a decrease in serum TAS associated with CAP ${ }^{35}$. However, there are few studies in the literature regarding the oxidative/antioxidant balance in children with CAP. In contrast to the findings of Katsoulis et al., we detected significantly higher TAS levels in CAP patients compared to healthy children. This discrepancy may be attributed to the difference in patient populations, as the study by Katsoulis et al. was conducted in adults, $63.3 \%$ of whom were smokers.

The currently available biomarkers WBC count and CRP reflect host inflammatory response to infection ${ }^{36}$. In our study, we found higher WBC and CRP levels in CAP patients as compared with controls, indicating that these classical inflammatory markers are still useful for the diagnosis of CAP in children.

MPO has a crucial role in fighting pathogens due to its virucidal and bactericidal properties. It is one of the enzymes whose expression serves as a suitable marker for inflammation that involves oxidative stress ${ }^{37}$. High blood or sputum MPO levels have been reported in lung diseases such as pneumonia, asthma, and cystic fibrosis ${ }^{3}, 38$. CLP is one of the non-enzymatic factors that may function as an antioxidant, and increased CLP level was previously demonstrated in pneumonia ${ }^{39,40}$. In this study, we wanted to investigate the role 
of both enzymatic and non-enzymatic factors in patients with CAP and determined that both MPO and CLP levels were higher in pediatric CAP patients than controls. Therefore, these variables also seem like good candidate biomarkers for the accurate diagnosis of pneumonia in children.

There are several potential limitations to our study. First, the small number of patients from a single center may limit the generalizability of the data. Second, the sampling time of the patients may not be synchronized according to the disease course without sequential blood sampling during the follow-up period of the patients. Thirdly, we did not analyze the results according to the etiological agent of pneumonia because the causative pathogen was not detected in many of our cases. Additionally, we did not evaluate any effect of any antioxidant medication in the present study. In infectious diseases, it has been shown that various inflammatory cells are activated, ROS are produced to fight against intracellular and extracellular microbes and this lead to increased potential antioxidant capacity ${ }^{4,18}$. It was reported that the use of antioxidants will greatly enhance the immune cell functions to control many bacterial and viral infections ${ }^{4}$. There may be changes in the antioxidant levels depending on the disease course and treatment. An interventional study also may be planned in near future. However this study is one of the few studies that evaluate the balance of oxidative and antioxidative stress biomarkers in children with CAP. Our findings, therefore, will shed light on the further investigators. Third, we did not analysed the results according to the etiological agent of pneumonia because of the lack of detection of the underlying causative agents of our cases.

In conclusion, parameters that provide information about a patient's oxidative state and antioxidant capacity, such as NT, TT, IMA, MPO, and CLP, may have diagnostic value in pediatric pneumonia. The acute-phase reactants WBC and CRP are still important conventional diagnostic and prognostic markers. Both thiol homeostasis parameters and IMA level seem likely to be influenced by disease severity. Our results suggest that plasma thiol levels and IMA may be good candidate biomarkers to predict the severity of pneumonia in children.

\section{References}

1. Harris M, Clark J, Coote N, et al. British Thoracic Society Standards of Care Committee. British Thoracic Society guidelines for the management of community acquired pneumonia in children: update 2011. Thorax 2011;356:1-23.

2. World Health Organization. Pneumonia. WHO, 2015.

3. J. Ciencewicki, S. Trivedi, S.R. Kleeberger. Oxidants and the pathogenesis of lung diseases. J Allergy Clin Immunol. 2008;122:456-470.

4. D. Morris, M. Khurasany, T. Nguyen et al. "Glutathione and Infection," Biochem Biopys Acta. 2013;1830:3329-3349.

5. Muravlyova L, Luchankiy-Molotov V, Bakirova R, Klyuyev D, Demidchik L, Lee V. Characteristics of the oxidative stress in blood of patients in depence of community-acquired pneumonia severity. Open Access Maced J Med Sci. 2016;4:122-127.

6. Principi N, Esposito S. Biomarkers in Pediatric Community-Acquired Pneumonia. Int J Mol Sci. 2017;18. pii: E447.

7. Haq IJ, Battersby AC, Eastham K, McKean M. Community acquired pneumonia in children. BMJ. 2017;356: j686.

8. Erel O, Neselioglu S. A novel and automated assay for thiol/disulphide homeostasis. Clinical Biochemistry $2014 ; 47: 326-332$.

9. Erel, O. A new automated colorimetric method for measuring total oxidant status. Clin. Biochem. 2005;38:1103-1111.

10. Erel O. A novel automated direct measurement method for total antioxidant capacity using a new generation, more stable ABTS radical cation. Clin Biochem 2004;37:277-85.

11. Harma, M., Harma, M. and Erel, O. Measurement of the total antioxidant response in preeclampsia with a novel automated method. Eur. J. Obstet. Gynecol. Reprod. Biol., 2005;118:47-51.

12. Eckerson HW, Romson J, Wyte C, La Du BN. The human serum paraoxonase polymorphism: identification of phenotypes by their response to salts. Am J Hum Genet. 1983;35:214-27. 
13. Góth L. A simple method for determination of serum catalase activity and revision of reference range. Clin Chim Acta. 1991;196:143-51.

14. Haagen L, Brock A. A new automated method for phenotyping arylesterase (EC 3.1.1.2) based upon inhibition of enzymatic hydrolysis of 4-nitrophenyl acetate by phenyl acetate. Eur J Clin Chem Clin Biochem. 1992;30:391-395.

15. Bradley PP, Priebat DA, Christensen RD, Rothstein G. Measurement of cutaneous inflammation: estimation of neutrophil content with an enzyme marker. J Invest Dermatol. 1982;78:206-209.

16. O. Erel, Automated measurement of serum ferroxidase activity, Clin. Chem. 1998;44:2313-2319.

17. Bar-Or D, Lau E, Winkler JV. A novel assay for cobalt-albumin binding and its potential as a marker for myocardial ischemia-a preliminary report. J Emerg Med. 2000;19:311-5.

18. Esen R, Aslan M, Kucukoglu ME et al. Serum paraoxonase activity, total thiols levels, and oxidative status in patients with acute brucellosis. Wien Klin Wochenschr. 2015;127:427-33.

19. Stocker R, Keaney Jr F. Role of oxidative modifications in atherosclerosis. Physiol Rev. 2004;84:1381478.

20. Biswas S, Chida AS, Rahman I. Redox modifications of protein-thiols: emerging roles in cell signaling. Biochem Pharmacol. 2006;71:551-64.

21. Gulpamuk B, Tekin K, Sonmez K et al. The significance of thiol/disulfide homeostasis and ischemiamodified albumin levels to assess the oxidative stress in patients with different stages of diabetes mellitus. Scand J Clin Lab Invest. 2018;78:136-142.

22. Kara SS, Erel O, Demirdag TB et al. Alteration of thiol-disulphide homeostasis in acute tonsillopharyngitis. Redox Rep. 2017;22:205-209.

23. Aycicek A, Iscan A, Erel O, Akcali M, Selek S. Total Antioxidant/Oxidant Status in Meningism and Meningitis. Pediatr Neurol. 2006;35:382-6.

24. Ozyazici S,Karateke F, Turan U et al. A Novel Oxidative Stress Mediator in Acute Appendicitis:Thiol/Disulphide Homeostasis Mediators Inflamm. 2016;2016:6761050.

25. Karadag-Oncel E, Erel O, Ozsurekci Y et al. Plasma oxidative stress and total thiol levels in CrimeanCongo hemorrhagic fever. Jpn J Infect Dis. 2014;67:22-26.

26. Parlak SE, Alisik M, Hezer H, Karalezli A, Hasanoglu HC, Erel O. Evaluation of dynamic thiol/disulfide redox state in community-acquired pneumonia. Saudi Med J. 2018;39:495-499.

27. Leelarungrayub J, Borisuthibandit T, Yankai A, Boontha K.Changes in oxidative stress from tracheal aspirates sampled during chest physical therapy in hospitalized intubated infant patients with pneumonia and secretion retention. Ther Clin Risk Manag. 2016;6:1377-1386.

28. Chan B, Dodsworth N, Woodrow J, et al. Sitespecific N-terminal auto-degradation of human serum albumin. Eur J Biochem 1995;227:524-528.

29. Cheng CW, Chien MH, Su SC, Yang SF. New markers in pneumonia. Clin Chim Acta. 2013;419:19-25.

30. Grover H, Saini R, Bhardwaj P, Bhardwaj A. Acute-phase reactans. Journal of Oral Research and Review. 2016:8;31-35.

31. Bolatkale M, Duger M, Ülfer G et al. A novel biochemical marker for community-acquired pneumonia: Ischemia-modified albumin. Am J Emerg Med. 2017;35:1121-1125.

32. Yerlikaya FH, Kurban S, Mehmetoglu I et al. Serum ischemia-modified albumin levels at diagnosis and during treatment of late-onset neonatal sepsis. J Matern Fetal Neonatal Med. 2014;27:1723-1727.

33. H.S. Park, S.R. Kim, Y.C. Lee. Impact of oxidative stress on lung diseases. Respirology. 2009;14:27-38.

34. Trefler S, Rodríguez A, Martín-Loeches I et al. Oxidative stress in immunocompetent patients with severe community-acquired pneumonia. A pilot study. Med Intensiva. 2014;38:73-82.

35. Katsoulis K, Kontakiotis T, Baltopoulos G, Kotsovili A, Legakis IN. Total antioxidant status and severity of communityacquired pneumonia: are they correlated? Respiration 2005;72:381-387.

36. Van den Bruel A, Thompson MJ, Haj-Hassan T. et al. Diagnostic value of laboratory tests in identifying serious infections in febrile children: systeMatic review. BMJ. 2011;8:d3082.

37. Pohanka M. Role of oxidative stress in infectious diseases. A review. Folia Microbiol (Praha). 2013;58:503-513.

38. Braun J, Dalhoff K, Lipp R et al. Myeloperoxidase, lactoferrin and elastase in bronchoalveolar lavage 
and plasma in pneumonia. Pneumologie. 1992;46:141-147.

39. Cemek M, Caksen H, Bayiroğlu F, Cemek F, Dede S. Oxidative stress and enzymic-non-enzymic antioxidant responses in children with acute pneumonia. Cell Biochem Funct. 2006;24:269-273.

40. Shepelenko AF, Khatskevich VL, Lishcheniuk OA, Korshunov GV, Dolmashkina MA. The role of humoral markers of inflammation activity in the evaluation of adequacy of antibacterial chemotherapy for community-acquired pneumonia. Voen Med Zh. 2005;326:25-30.
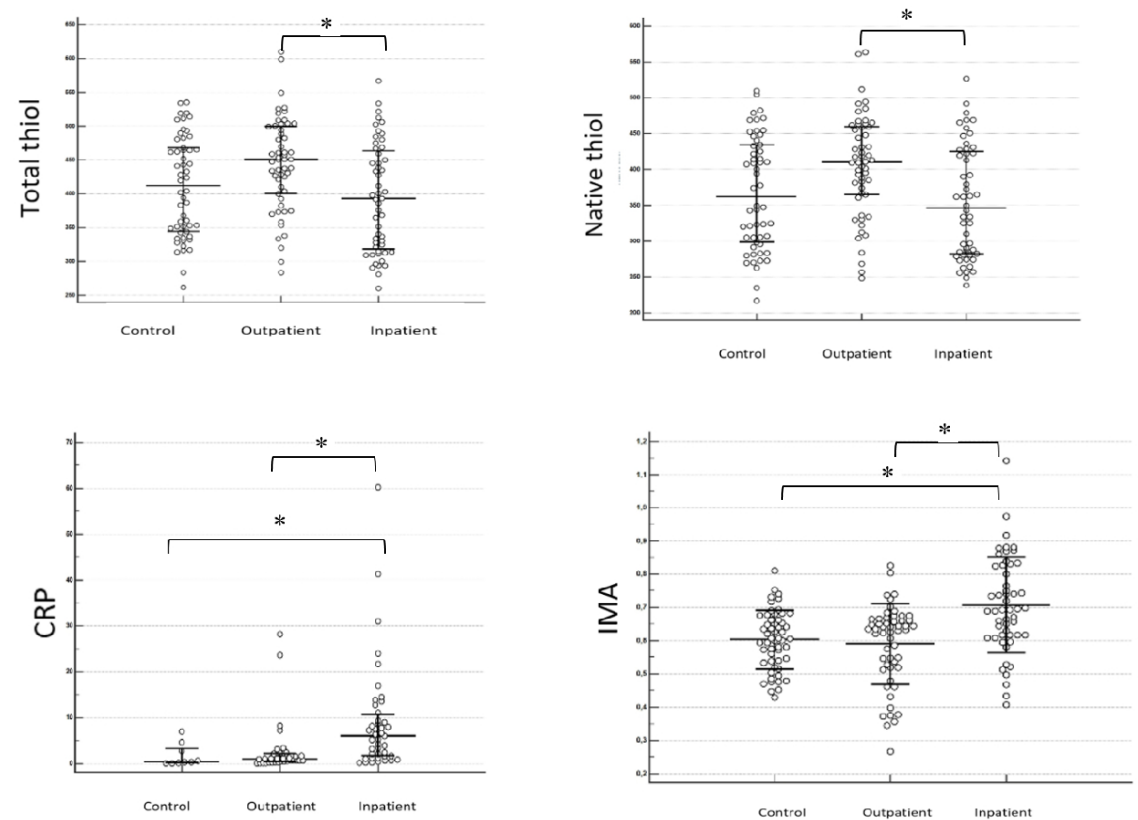

Fig 1. Comparison of serum thiols, CRP and IMA levels in patients with pneumonia and healthy controls. Groups were compared using Kruskal-Wallis test and values were considered statistically significant $(*)$ when $p<0.05$. 


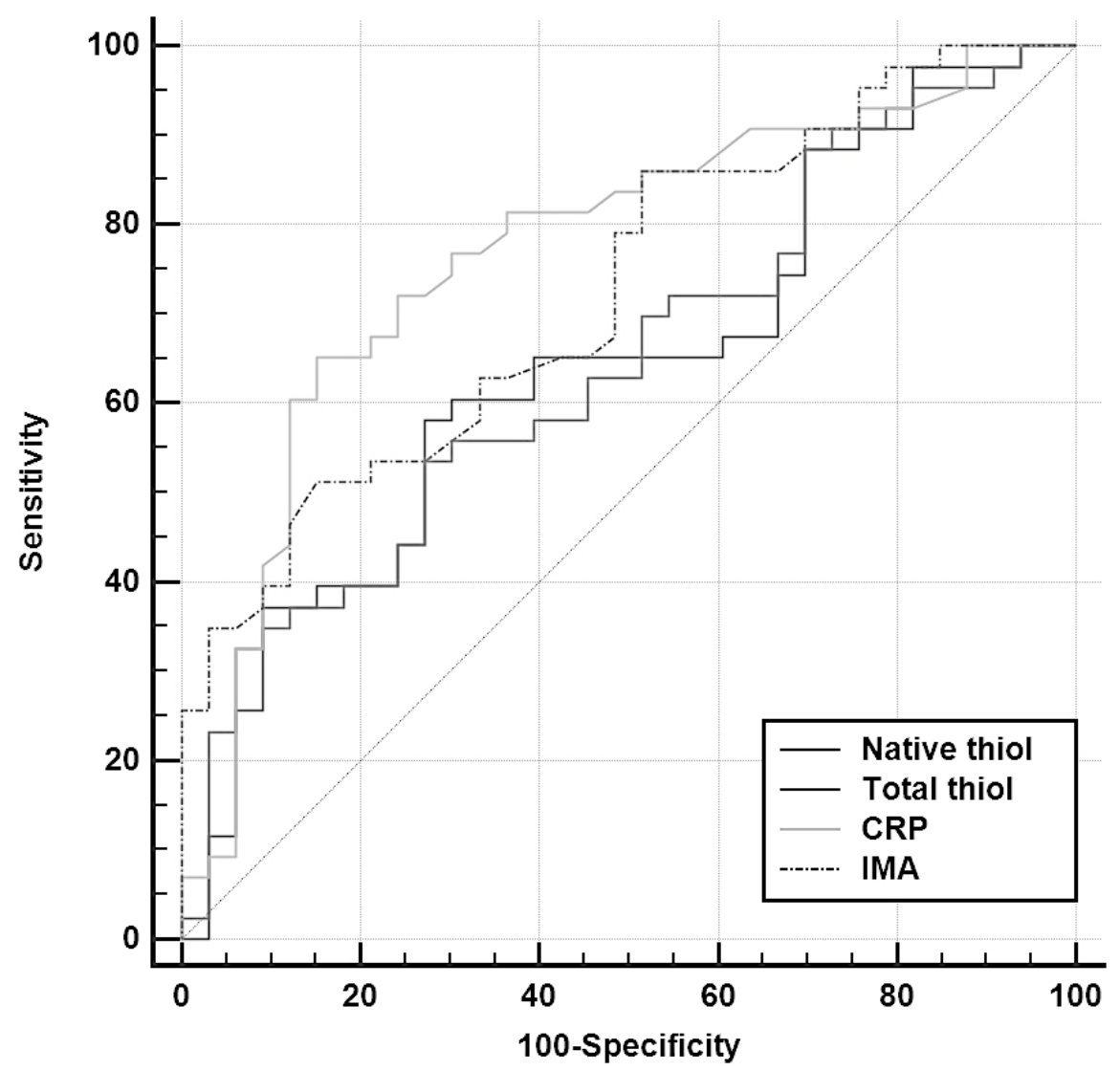

\section{Hosted file}

table 1.docx available at https://authorea.com/users/337442/articles/481571-oxidant-andantioxidant-balance-in-children-with-community-acquired-pneumonia 